\title{
Production of hardened body by direct bonding of sand particles
}

\author{
Yuya SAKAI ${ }^{*}$ and Ahmad FARAHANI ${ }^{2}$
}

1 Institute of Industrial Science, The University of Tokyo, 4-6-1 Komaba, Meguro, Tokyo 153-8505, Japan

2 Department of Civil Engineering, Graduate School of Engineering, The University of Tokyo, 4-6-1

Komaba, Meguro, Tokyo 153-8505, Japan

*Corresponding author (ysakai@iiis.u-tokyo.ac.jp)

*This is the transaction of the following note published in Seisan Kenkyu, Vol. 75, 2021 as "Production of Hardened Body by Direct Bonding of Sand Particles."

\begin{abstract}
Raw materials used for producing concrete, such as sand and gravel, are in short supply worldwide. It has been reported that the production of cement, one of the main constituents of concrete, results in the emission of a large amount of $\mathrm{CO}_{2}$, accounting for $8 \%$ of total global $\mathrm{CO}_{2}$ emissions. In this study, we developed a method to produce a construction material using only sand as the solid raw material. By utilizing a technique to produce organic silicon material from sand, we placed sand, alcohol, and catalyst in a closed vessel and heated it to $240{ }^{\circ} \mathrm{C}$. After $24 \mathrm{~h}$, the sand particles were bonded to each other, and a hardened body was formed. A tradeoff in production conditions, such as temperature, time, and the amount of sand, was confirmed.
\end{abstract}

\section{Introduction}

Concrete is one of the most important construction materials, and its global production amount is increasing, with most contribution from developing countries. Concrete is formed using cement, sand, gravel, and water, and the main raw material of cement is limestone. Recently, in many countries and regions, the above materials are diminishing. For example, India is expected to become the largest cement-producing country, overtaking China shortly; however, good-grade limestone is depleting ${ }^{1}$. Sand for concrete is also exhausting worldwide ${ }^{2)}$ and is required to have a specific size distribution; therefore, desert sand because of its extremely small size and high uniformity is unsuitable to be used in construction. Saudi Arabia is a country whose $98 \%$ of national land is desert; however, sand for construction is in short supply, and its export was tentatively stopped in 2009. In addition to the deficiency of concrete raw materials, $\mathrm{CO}_{2}$ emission is another problem in the concrete industry. In the production of cement, limestone, mainly $\mathrm{CaCO}_{3}$, is combusted at $1450{ }^{\circ} \mathrm{C}$, which consequently generates $8 \%$ of the total global $\mathrm{CO}_{2}$ emissions $^{3)}$. 
The author has developed new construction materials to deal with the deficiency of concrete raw materials and reduce the $\mathrm{CO}_{2}$ emissions from the construction industry. For example, utilization of concrete waste is one of the most urgent issues in the construction industry; thus, we crushed concrete waste into a powder, which was compacted for recycling without the addition of new materials and without producing byproducts ${ }^{4}$. Depending on the compaction conditions and the treatment after the compaction, the recycled concrete can present a higher strength than the original concrete. In another study, instead of using cement, wood was used as the adhesive for concrete waste or sand and gravel to produce a new construction material ${ }^{5)}$, ${ }^{6}$. By heat pressing a mixture of these materials at approximately $200^{\circ} \mathrm{C}$, botanical concrete is produced, whose bending strength can be seven times higher than that of normal concrete. These methods do not require new raw materials, and $\mathrm{CO}_{2}$ emissions can be significantly reduced. However, in the first method, a hardened cement paste flows under pressure, filling the gaps among the particles and acting as an adhesive; therefore, concrete waste is necessary as a material. As seen in Figure 1, compacting silica sand even at a very high pressure (300 MPa) does not produce adhesion among the sand particles. The second method requires wood or plant; therefore, this technique is inapplicable in regions with scarce wood or plants, such as deserts, which account for one-fourth of the land area on the earth, and high mountain areas.

Low-quality sand, which is currently not used for conventional constructions, is practically inexhaustible and available worldwide. Therefore, if construction materials could be produced from low-quality sand, such as desert sand, then exhaustion of raw materials will not be problematic. On earth, the main component of sand is $\mathrm{SiO}_{2}$, which is the same on the moon ${ }^{7)}$ and $\mathrm{Mars}^{8}$. Therefore, the new technique can be applied in space development.

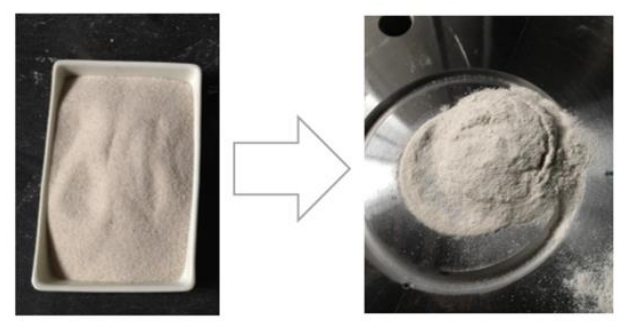

Figure 1 Compaction of silica sand at $300 \mathrm{MPa}$

The simplest method to produce construction materials from sand is to heat sand to its melting point and subsequently cool it. However, the melting point of $\mathrm{SiO}_{2}$ is very high, $1650{ }^{\circ} \mathrm{C}$, which is unsuitable for producing eco-friendly materials. Fukaya et al. ${ }^{9}{ }^{9}$ developed a method to produce tetraalkoxysilane $\left(\mathrm{Si}(\mathrm{OR})_{4}\right)$ from sand $\left(\mathrm{SiO}_{2}\right)$ using an alcohol $(\mathrm{ROH})$ and a catalyst, as expressed below:

$$
\mathrm{SiO}_{2}+4 \mathrm{ROH} \rightleftharpoons \mathrm{Si}(\mathrm{OR})_{4}+2 \mathrm{H}_{2} \mathrm{O} .
$$

In the above equation, removing water $\left(\mathrm{H}_{2} \mathrm{O}\right)$ pushes the reaction from left to right, producing more tetraalkoxysilane efficiently. Here, if we interpret the rightward reaction as a breaking of the chemical 
bonds in sand and the leftward reaction as the formation of chemical bonds to form sand, there is a possibility that maintaining water in the reaction will bond the sand particles by a repetitive bond breaking and formation.

In this study, a basic experiment was performed to determine whether sand particles can be bonded to each other by the above approach; to this end, the experimental conditions were varied, such as the amount of alcohol and catalyst, heating temperature, and duration.

\section{Experimental method}

In this study, silica sand (No. 8, smaller than $0.2 \mathrm{~mm}$ in diameter), 2,2-dimethoxypropane (Wako first grade, FUJIFILM Wako Pure Chemical Corporation), ethanol (95) (special grade reagent, FUJIFILM Wako Pure Chemical Corporation), and potassium hydroxide (KOH) (special grade reagent, FUJIFILM Wako Pure Chemical Corporation) were used. The dehydration of 2,2-dimethoxypropane was conducted in an experiment by Fukaya et al. ${ }^{9}$. . The experimental conditions are listed in Table 1. In the experiment, first, a copper foil was folded to form a container with a square area of $4 \mathrm{~cm}^{2}$, as shown in Fig. 2, and silica sand was placed in it. This copper container was placed in a reaction vessel $(100 \mathrm{~mL})$, and the chemicals were placed into the vessel and sealed. Sand was not directly placed in the vessel, instead it was placed in a copper container to avoid the difficulty in removing the hardened sample in case of bonding among the sand particles.

Table 1 Experimental conditions

\begin{tabular}{l|l}
\hline $\mathrm{KOH}$ & $0.33-1.32 \mathrm{~g}$ \\
Silica sand & $2-8 \mathrm{~g}$ \\
Ethanol & $50,75 \mathrm{~mL}$ \\
2,2-Dimethoxypropane & $0.0-2.4 \mathrm{~mL}$ \\
Temperature & $180-243^{\circ} \mathrm{C}$ \\
Duration & $24-72 \mathrm{~h}$ \\
\hline
\end{tabular}

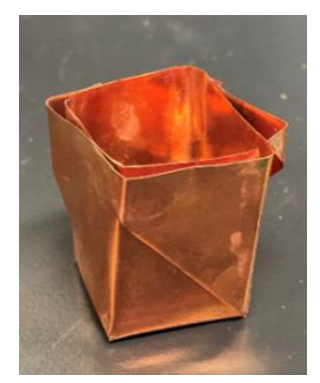

Figure 2 Container formed by folding copper foil 
The experimental setup is shown in Fig. 3. The vessel is heated using a band heater placed around the vessel. A pressure gauge and a safety valve of $20 \mathrm{MPa}$ are connected to the vessel. A deep hole is made from the cap of the vessel to its inside, and a thermocouple is inserted in the hole to measure the temperature of the contents in the vessel under a sealed condition. The thermocouple and the band heater are connected to a control system to measure and control the temperature of the contents by adjusting the output of the band heater. Further, the temperature of the contents is set as $243{ }^{\circ} \mathrm{C}$, and the heating duration is $24 \mathrm{~h}$.

The objective of this study is to determine whether sand particles can be bonded using an alcohol and a catalyst. Therefore, after production, solid formation was examined. In case of solid formation, despite being a qualitative evaluation, its ability to bear the load applied by fingers was evaluated.

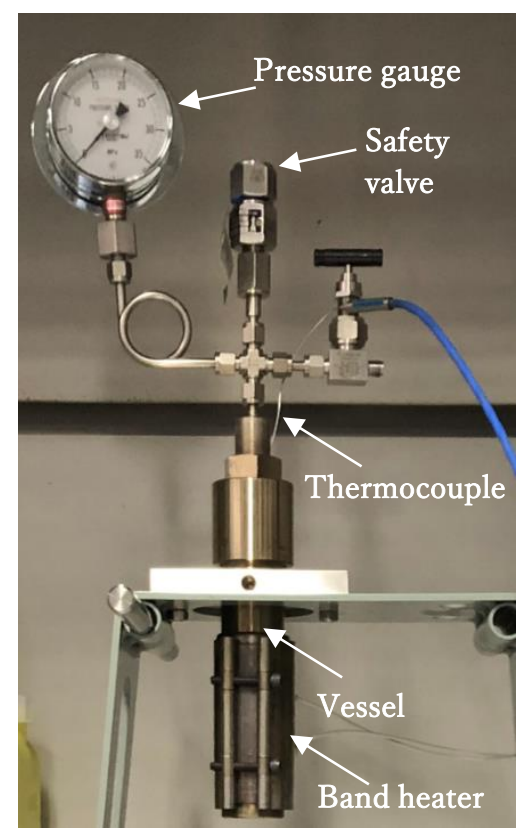

Figure 3 Experimental setup

\section{Results and discussions}

The treatment conditions and the results are listed in Table 2. In the "Results" column in the table, "C" indicates no bonding among the sand particles, and " $\mathrm{B}$ " indicates that a solid is formed but broken easily. In comparison, "A" corresponds to a solid with sufficiently high strength against compression by fingers. An example of the products determined as " $A$ " is shown in Fig. 4. Examples of other products are shown in Appendix. 
Table 2 Treatment conditions and results

\begin{tabular}{|c|c|c|c|c|c|c|c|}
\hline Case & $\begin{array}{c}\mathrm{KOH} \\
\mathrm{g} \\
\end{array}$ & $\begin{array}{c}\text { Sand } \\
\mathrm{g} \\
\end{array}$ & $\begin{array}{c}\text { Ethanol } \\
\text { mL }\end{array}$ & $\begin{array}{c}\text { 2,2-Dimethoxy } \\
\text { propane } \\
\mathrm{mL} \\
\end{array}$ & $\begin{array}{c}\text { Temperature } \\
{ }^{\circ} \mathrm{C} \\
\end{array}$ & $\begin{array}{c}\text { Duration } \\
\mathrm{h} \\
\end{array}$ & Results \\
\hline 1 & 0.66 & 4.0 & 75 & 0.0 & 243 & 24 & $\mathrm{~B}$ \\
\hline 2 & 0.66 & 4.0 & 75 & 0.9 & 243 & 24 & $\mathrm{~A}$ \\
\hline 3 & 0.33 & 2.7 & 50 & 0.15 & 243 & 24 & $\mathrm{C}$ \\
\hline 4 & 0.33 & 2.4 & 50 & 0.15 & 243 & 24 & $\mathrm{~B}$ \\
\hline 5 & 0.33 & 2.0 & 50 & 0.15 & 243 & 24 & A \\
\hline 6 & 0.33 & 2.7 & 50 & 0.15 & 243 & 36 & $\mathrm{~B}$ \\
\hline 7 & 0.33 & 2.4 & 50 & 0.15 & 243 & 36 & A \\
\hline 8 & 0.33 & 2.7 & 50 & 0.15 & 243 & 48 & A \\
\hline 9 & 0.33 & 4.0 & 75 & 0.3 & 243 & 24 & $\mathrm{~B}$ \\
\hline 10 & 1.32 & 4.0 & 75 & 0.3 & 243 & 24 & A \\
\hline 11 & 0.33 & 2.0 & 50 & 0.3 & 243 & 24 & A \\
\hline 12 & 0.66 & 4.0 & 50 & 0.3 & 243 & 24 & A \\
\hline 13 & 0.99 & 6.0 & 50 & 0.3 & 243 & 24 & $\mathrm{C}$ \\
\hline 14 & 1.32 & 8.0 & 50 & 0.3 & 243 & 24 & $\mathrm{C}$ \\
\hline 15 & 0.66 & 4.0 & 75 & 0.4 & 243 & 24 & $\mathrm{C}$ \\
\hline 16 & 0.66 & 4.0 & 75 & 0.6 & 243 & 24 & $\mathrm{C}$ \\
\hline 17 & 0.66 & 4.0 & 75 & 0.8 & 243 & 24 & $\mathrm{~B}$ \\
\hline 18 & 0.66 & 4.0 & 75 & 1.0 & 243 & 24 & $\mathrm{~B}$ \\
\hline 19 & 0.66 & 4.0 & 75 & 1.2 & 243 & 24 & $\mathrm{~A}$ \\
\hline 20 & 0.66 & 4.0 & 75 & 1.4 & 243 & 24 & A \\
\hline 21 & 0.66 & 4.0 & 75 & 1.6 & 243 & 24 & A \\
\hline 22 & 0.66 & 4.0 & 75 & 1.8 & 243 & 24 & $\mathrm{~B}$ \\
\hline 23 & 0.66 & 4.0 & 75 & 2.0 & 243 & 24 & $\mathrm{~B}$ \\
\hline 24 & 0.66 & 4.0 & 75 & 2.4 & 243 & 24 & $\mathrm{C}$ \\
\hline 25 & 0.66 & 2.0 & 75 & 0.5 & 243 & 24 & A \\
\hline 26 & 0.66 & 4.0 & 75 & 0.9 & 243 & 24 & A \\
\hline 27 & 0.66 & 6.0 & 75 & 1.4 & 243 & 24 & $\mathrm{C}$ \\
\hline 28 & 0.66 & 8.0 & 75 & 1.8 & 243 & 24 & $\mathrm{C}$ \\
\hline 29 & 0.66 & 4.0 & 75 & 0.6 & 180 & 24 & $\mathrm{C}$ \\
\hline 30 & 0.66 & 4.0 & 75 & 0.6 & 200 & 24 & $\mathrm{~B}$ \\
\hline 31 & 0.66 & 4.0 & 75 & 0.6 & 220 & 24 & A \\
\hline 32 & 0.66 & 4.0 & 75 & 0.6 & 243 & 24 & A \\
\hline 33 & 0.66 & 4.0 & 75 & 0.6 & 200 & 72 & $\mathrm{~A}$ \\
\hline
\end{tabular}




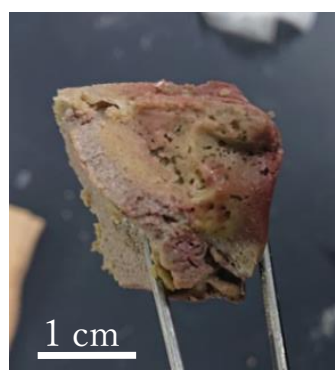

Figure 4 Example of products

In Cases 1 and 2, the effect of 2,2-dimethoxypropane was studied. The sample with 2,2dimethoxypropane is hardened well (A) but the one without 2,2-dimethoxypropane (B) is not. This result indicates that the dehydration reaction by 2,2-dimethoxypropane is effective for bonding and that removal of a certain amount of water may be necessary.

In Cases 3-8, the amount of sand and the heating duration were varied. Table 2 shows the trend that a large amount of sand produces poor bonding ( $\mathrm{B}$ or $\mathrm{C}$ ). However, even when the amount of sand is large, a long duration of heating produces a strong solid (A). Therefore, to produce a large solid with a large amount of sand, a longer duration is required. Specifically, to produce a strong solid and reduce the heating duration, reducing the amount of sand will be effective.

The effect of $\mathrm{KOH}$ was studied in Cases 2, 9, and 10. A small amount of $\mathrm{KOH}$ does not provide sufficient strength (B); therefore, a certain amount of $\mathrm{KOH}$ may be necessary for the bonding. However, in Cases 11-14, the amounts of sand and $\mathrm{KOH}$ were varied while maintaining their weight ratio. Even though the weight ratio was the same, solid production with a large amount of sand (C) failed. By comparing Cases 9 and 12 in which the amounts of sand are the same, reduction in ethanol produced a weaker solid (B) although more $\mathrm{KOH}$ was added. These results indicate that not only the weight ratio of sand and $\mathrm{KOH}$ but also the absolute amounts of sand, ethanol, and 2,2dimethoxypropane are important.

In Cases 15-24, the amount of 2,2-dimethoxypropane was varied. Solid samples were produced when the amount of 2,2-dimethoxypropane was within a certain range, indicating that excessive 2,2dimethoxypropane may remove excessive water and inhibit the bonding. In Cases 25-28, the amounts of sand and 2,2-dimethoxypropane were varied while maintaining their proportion. Although the proportion was the same, a larger amount of sand does not produce a solid (C); therefore, it was reconfirmed that not only the proportion of sand and $\mathrm{KOH}$ but also the amounts of sand, ethanol, and 2,2-dimethoxypropane are important.

Finally, in Cases 29-33, the temperature was varied, and it was found that a lower temperature yields a weaker solid (C or B). Fukaya et al. ${ }^{9}$ reported a similar tendency: a lower temperature caused a smaller production of tetraalkoxysilane. These tendencies are attributed to a lower temperature reducing the reaction rate. For example, as seen in Case 33 , even at $200^{\circ} \mathrm{C}$, heating for $72 \mathrm{~h}$ yielded a 
strong solid (A).

The results obtained in this study revealed that sand particles can be bonded to each other using an alcohol and a catalyst. To use this solid extensively as a substitute material for concrete, in a future study, it is necessary to confirm that the solid has sufficient strength and durability and the heating temperature and duration are reduced, and materials that are alternatives for sand need to be used repeatedly. In addition, the produced bonds among the sand particles need to be studied to understand the bonding mechanism.

\section{Conclusions}

In this study, the possibility of bonding sand particles to each other directly using an alcohol and a catalyst is examined. The obtained results are summarized as follows:

- By mixing sand, an alcohol, and a catalyst, a solid composed of sand particles is produced.

- There is a trade-off between the heating temperature and duration in the production. Specifically, when the amount of sand is large or the heating temperature is low, a longer heating can compensate for the strength.

\section{Acknowledgement}

The authors would like to thank Dr. Fukaya Norihisa from the Interdisciplinary Research Center for Catalytic Chemistry, National Institute of Advanced Industrial Science and Technology for useful comments.

\section{References}

1) HKN Bhattacharjee and GC Mishra: Utilisation of high silica low grade limestone, Indian Cement Review Magazine, Oct. 2018

2) Vince Beiser: The world in a grain, Riverhead Books, 2018

3) R.M. Andrew: Global $\mathrm{CO}_{2}$ emissions from cement production, Earth System Science Data Discussions, pp. 195-217, 2018

4) Yuya Sakai, Biruktawit Taye Tarekegne and Toshiharu Kishi: Recycling of hardened cementitious material by pressure and control of volumetric change, Journal of Advanced Concrete Technology, Vol. 14, No. 2 pp. 47-54, 2016

5) Liang Li and Yuya Sakai: Experimental Study of the Bending Strength of Recycled Concrete and Wooden Waste by Heating Compaction, The Sixth International Conference on Construction Materials (ConMat'20), Fukuoka, Japan, 2020

6) Liang Li: Recycling of concrete waste with wood waste through heating compaction, Master's thesis submitted to KTH royal institute of technology, Sweden, 2020

7) Papike JJ, Simon SB \& Laul JC: The lunar regolith: Chemistry, mineralogy, and petrology, 
Reviews of Geophysics, Vol. 20, pp. 761-826, 1982

8) Material Safety Data Sheet of JSC Mars-1A Martian Regolith Simulant, Orbital Technologies Corporation, 2008

9) Norihisa Fukaya, Seong Jib Choi, Toshio Horikoshi, Hiroshi Kumai, Minoru Hasegawa, Hiroyuki Yasuda, Kazuhiko Sato, and Jun-Chul Choi: Synthesis of Tetramethoxysilane from Silica and Methanol Using Carbon Dioxide and an Organic Dehydrating Reagent, Chemistry Letters 2016 45:7, 828-830

\section{Appendix}

The photos made from various types of sand.

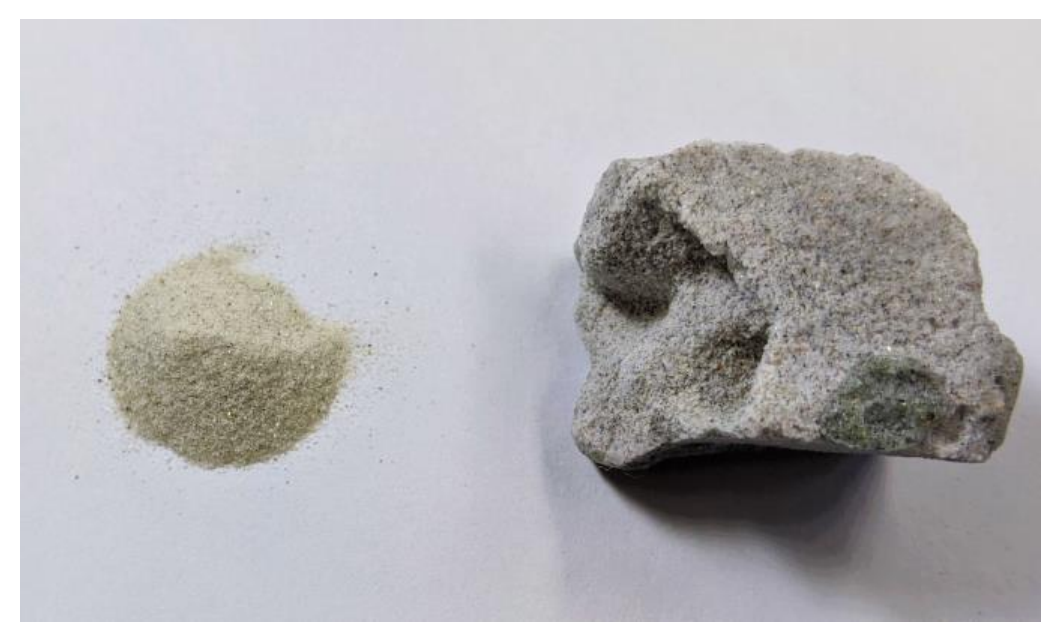

Appendix Fig. 1 A solid made from silica sand

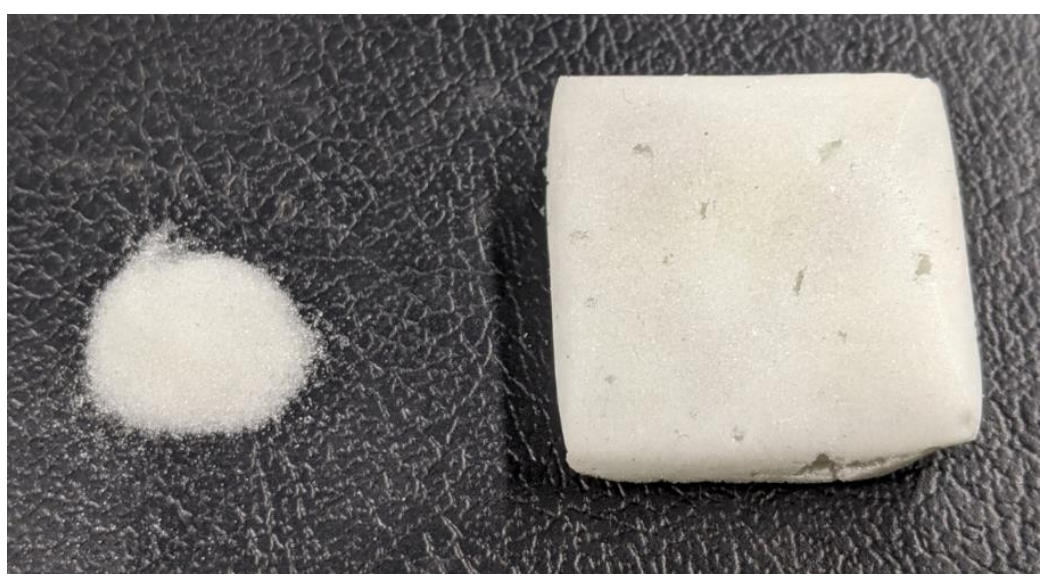

Appendix Fig. 2 A solid made from glass beads 


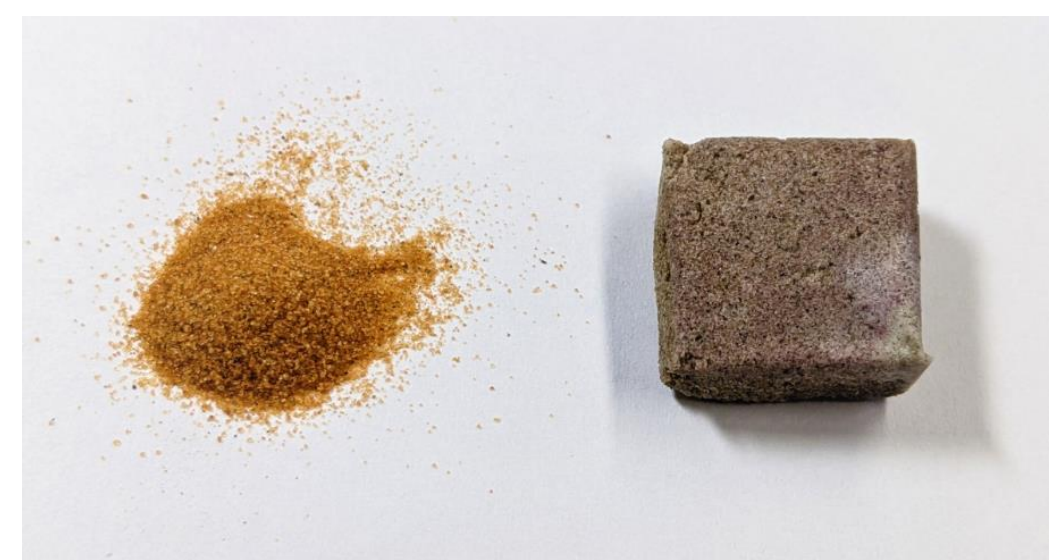

Appendix Fig. 3 A solid made from Namib desert sand

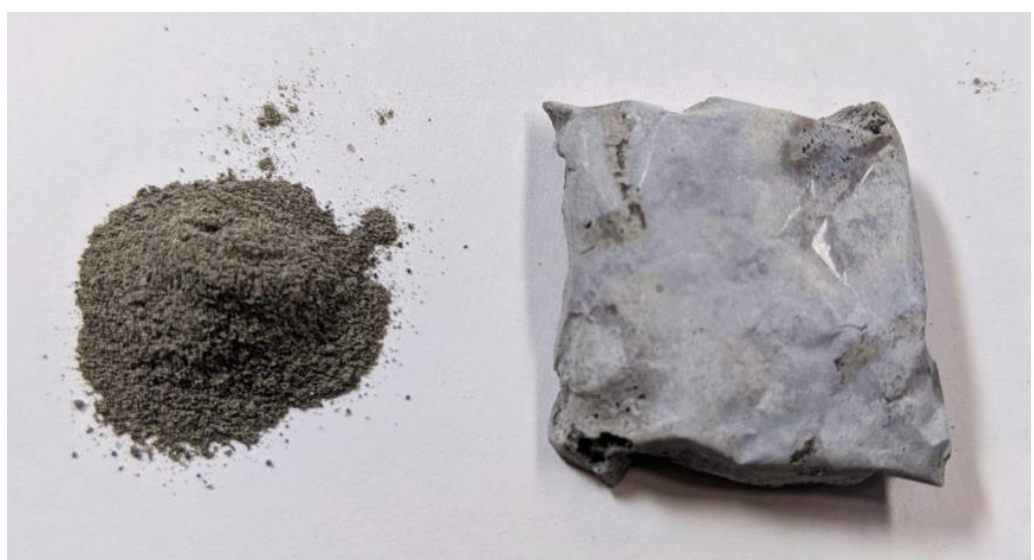

Appendix Fig. 4 A solid made from simulated sand of the moon (The sand is supplied from NICHIREKI CO.,LTD., Tokyo, Japan) 